\title{
Long-term Combination Therapy With a-Blockers and 5a-Reductase Inhibitors in Benign Prostatic Hyperplasia: Patient Adherence and Causes of Withdrawal From Medication
}

\author{
Hueih Ling Ong ${ }^{1}$, Chun-Hou Liao ${ }^{2}$, Hann-Chorng Kuo ${ }^{1}$ \\ ${ }^{1}$ Department of Urology, Buddhist Tzu Chi General Hospital and Tzu Chi University, Hualien, Taiwan \\ ${ }^{2}$ Division of Urology, Department of Urology, Cardinal Tien Hospital and School of Medicine, Fu-Jen Catholic University, New Taipei City, Taiwan
}

Purpose: To investigate long-term therapeutic effects and patient adherence to a combination therapy of a $5 a$-reductase inhibitor and an $\alpha$-blocker and to identify causes of withdrawal from medication in patients with clinical benign prostatic hyperplasia (BPH).

Methods: BPH patients with lower urinary tract symptoms (LUTS) receiving combination therapy with follow-ups for 1-12 years were retrospectively analyzed. Therapeutic effects were assessed at baseline and annually by measuring International Prostatic Symptoms Score, quality of life index, total prostate volume (TPV), maximal flow rate, voided volume, postvoid residual volume and prostate-specific antigen level. Causes of discontinued combination therapy were also investigated.

Results: A total of 625 patients, aged 40-97 years (mean, 73 years) were retrospectively analyzed. All measured parameters showed significant improvements after combination therapy. Three hundred sixty-nine patients (59\%) discontinued combination therapy with a mean treatment duration of 2.2 years. The most common reasons for discontinued treatment were changing medication to monotherapy with a-blockers or antimuscarinics (124 patients, 19.8\%), receiving surgical intervention (39 patients, 6.2\%), and LUTS improvement (53 patients, 8.5\%). Only 64 patients (10.2\%) were loss to follow-up and 6 (1.0\%) discontinued combined treatment due to adverse effects. Smaller TPV after short-term combination treatment caused withdrawal from combination therapy.

Conclusions: BPH patients receiving long-term combination therapy showed significant improvement in all measured parameters. Changing medication, improved LUTS and choosing surgery are common reasons for discontinuing combination herapy. A smaller TPV after short-term combination treatment was among the factors that caused withdrawal from combination therapy.

Keywords: Adrenergic alpha-1 Receptor Antagonists; 5-alpha Reductase Inhibitor; Medication Adherence; Adverse Effects

- Research Ethics: This study had been approved by the Ethics Committee of Buddhist Tzu Chi General Hospital, Hualien, Taiwan (approval number: 102-85). Informed consent was waived by the Ethics Committee of the Buddhist Tzu Chi General Hospital, Hualien, Taiwan, as the chart review involved a regular treatment and the study was retrospectively performed.

- Conflict of Interest: No potential conflict of interest relevant to this article was reported.

\section{INTRODUCTION}

Benign prostatic hyperplasia $(\mathrm{BPH})$ is a progressive disease commonly associated with bothersome lower urinary tract symptoms (LUTS). It may result in complications, such as acute urinary retention, and require BPH-related surgery [1-3]. Treat-

Corresponding author: Hann-Chorng Kuo (iD http://orcid.org/0000-0001-7165-4771 Department of Urology, Buddhist Tzu Chi General Hospital, 707, Section 3, Chung Yang Road, Hualien, Taiwan

E-mail: hck@tzuchi.com.tw / Tel: +886-3-8561825 (ext. 2117) /

(i) $\$$ This is an Open Access article distributed under the terms of the Creative Commons Attribution Non-Commercial License (http://creativecommons.org/licenses/by-nc/4.0/) which permits unrestricted non-commercial use, distribution, and reproduction in any medium, provided the original work is properly cited.

Fax: +886-3-8560794

Submitted: February 5, 2016 / Accepted after revision: February 19, 2016 
ments with $\alpha-1$ blockers have been found to rapidly improve the maximal flow rate (Qmax) and quality of life index (QoL-I) $[4,5]$. Previous trials have revealed that $5 \alpha$-reductase inhibitors (5ARI), such as finasteride or dutasteride, could reduce the total prostate volume (TPV) and surgical risk in long-term follow-ups [6,7]. Combination therapy with a-blockers and 5ARIs has been proven effective in reducing LUTS, decreasing TPV, and reducing the risk of disease progression compared to treatment with a single medication or placebo [8,9]. Combination therapy with $a$-blockers and 5ARI has been recommended for patients with moderate-to-severe LUTS and enlarged prostates in the guidelines for BPH/LUTS management [10,11].

BPH with LUTS is not life threatening and life-long medical treatment has become the main management strategy in recent decades [10]. Long-term reports on patient compliance with combination treatment for LUTS due to BPH are scarce. This study aims to assess the long-term therapeutic effects of combination therapy at a single center in Hualien County, Taiwan. The causes of discontinuing one or both medications were also assessed.

\section{MATERIALS AND METHODS}

Patients with clinical BPH (TPV $\geq 30 \mathrm{~mL}$ ) receiving combination treatment for at least 1 year were retrospectively analyzed. All patients were treated with combined 5ARI (dutasteride 0.5 $\mathrm{mg}$ once a day) and alpha-blockers (tamsulosin $0.4 \mathrm{mg}$ or doxazosin $4 \mathrm{mg}$ once a day) from the beginning and had regular follow-ups. These patients were investigated for LUTS, prostate indicators, and uroflowmetry parameters annually after starting combination treatment. Data were retrospectively collected between 2003 and 2015. The inclusion criteria were male sex, aged 40 and over, a diagnosis of BPH associated with LUTS, and first prescription of a combination of an $\alpha-1$ blocker and a 5ARI for at least one year. Patients with neurological lesions, recurrent urinary tract infection, and prostate cancer confirmed by biopsy were excluded from this study. This study had been approved by the Ethics Committee of the Buddhist Tzu Chi General Hospital, Hualien, Taiwan (approval number: 102-85). Informed consent was waived by the Ethics Committee of the Buddhist Tzu Chi General Hospital, Hualien, Taiwan as the chart review involved a regular treatment and the study was retrospectively performed.

Routine clinical assessments of BPH at baseline and annual follow-ups included digital rectal examination, International
Prostatic Symptom Score (IPSS), QoL-I measurement, transrectal ultrasound of the TPV and transition zone index (TZI), uroflowmetry (Qmax, voided volume [VoL] and postvoid residual [PVR]), and prostate-specific antigen (PSA). Baseline data were collected every 6 months for 2 years and then annually, starting from the time the patient was enrolled in the study until the discontinuation of combination treatment. If patients died or did not refill medication, they were considered to have dropped-out from the follow-up analysis. If medications were changed during the follow-up period, patients were grouped into the discontinued medication group and the parameters were not included in the follow-up analysis. Reasons for discontinuing treatment were determined through a face-to-face interview or telephone call by the same research assistant. Patient characteristics and parameters which might be associated with discontinuation of combination treatment were also analyzed.

In the analyses, the mean and standard deviations were calculated for the continuous variables, and numbers and percentages for categorical data. Continuous data of therapeutic outcomes were compared between the groups at different timepoints. Paired t-tests and Pearson chi-square tests were used to compare the measured parameters from baseline to each time point, and the relationship between QoL-I and adherence to medication as appropriate. A P-value of less than 0.05 was considered statistically significant.

\section{RESULTS}

A total of 625 patients were recruited for this study. The mean age at baseline was $72.9 \pm 9.0$ years (range, $40-97$ years). The patient number at each follow-up year is listed in Table 1 . The mean treatment duration of the recruited patients was $3.16 \pm 2.94$ years (range, 1-12 years). There were 256 patients (41\%) who continued taking combination therapy, with a mean treatment duration of $4.59 \pm 3.34$ years (range, 1-12 years), whereas 369 patients (59\%) discontinued combination therapy, with a mean treatment duration of $2.20 \pm 2.16$ years (range, $1-12$ years).

\section{Changes in Parameters From Baseline to Different Time- Points in Patients Who Received Combination Therapy}

From baseline to the longest period of 12 years of ongoing treatment, combination therapy showed sustained and continued improvements in all parameters. In general, improvement became less prominent after 6 years of follow-ups. The distribu- 
Table 1. The parameters of each time-point after combination therapy along 12 years of period

\begin{tabular}{|c|c|c|c|c|c|c|c|c|}
\hline Year & IPSS-T & QoL-I & Qmax (mL/sec) & $\mathrm{Vol}(\mathrm{mL})$ & $\operatorname{PVR}(\mathrm{mL})$ & $\mathrm{TPV}(\mathrm{mL})$ & TZI & PSA (ng/mL) \\
\hline Baseline & $13.5 \pm 8.11$ & $3.23 \pm 1.3$ & $10.01 \pm 4.76$ & $190.0 \pm 118.8$ & $76.4 \pm 79.8$ & $50.0 \pm 23.5$ & $0.46 \pm 0.14$ & $4.21 \pm 5.40$ \\
\hline $1(\mathrm{n}=624)$ & $7.45 \pm 5.60^{*}$ & $2.15 \pm 0.85^{*}$ & $11.5 \pm 5.69^{*}$ & $219.6 \pm 132.9^{*}$ & $67.5 \pm 71.7^{*}$ & $44.5 \pm 22.1^{*}$ & $0.44 \pm 0.14^{*}$ & $2.69 \pm 3.29^{*}$ \\
\hline $2(\mathrm{n}=536)$ & $6.58 \pm 5.01^{*}$ & $2.12 \pm 0.75^{*}$ & $12.1 \pm 5.88^{*}$ & $238.8 \pm 145.1^{*}$ & $62.3 \pm 63.3^{*}$ & $45.4 \pm 22.3^{*}$ & $0.42 \pm 0.13^{*}$ & $2.78 \pm 3.94^{*}$ \\
\hline $3(\mathrm{n}=398)$ & $6.60 \pm 4.89^{*}$ & $2.05 \pm 0.66^{*}$ & $12.4 \pm 6.15^{*}$ & $246.7 \pm 146.8^{*}$ & $59.3 \pm 62.7^{*}$ & $47.9 \pm 22.9^{*}$ & $0.44 \pm 0.13^{*}$ & $3.43 \pm 7.00^{*}$ \\
\hline $4(\mathrm{n}=265)$ & $6.34 \pm 5.20^{*}$ & $2.02 \pm 0.66^{*}$ & $12.9 \pm 5.99^{*}$ & $236.3 \pm 132.9^{*}$ & $64.14 \pm 70.5^{*}$ & $48.8 \pm 23.3^{*}$ & $0.44 \pm 0.122^{*}$ & $3.12 \pm 5.35^{*}$ \\
\hline $5(\mathrm{n}=221)$ & $5.75 \pm 4.62^{*}$ & $1.98 \pm 0.63^{*}$ & $12.5 \pm 5.74^{*}$ & $239.7 \pm 142.1^{*}$ & $62.16 \pm 69.7^{*}$ & $47.5 \pm 21.1$ & $0.43 \pm 0.124^{*}$ & $2.29 \pm 2.75^{*}$ \\
\hline 186) & $6.19 \pm 4.68^{*}$ & $2.02 \pm 0.68^{*}$ & $11.96 \pm 5.17^{*}$ & $229.1 \pm 148.4^{*}$ & $53.3 \pm 63.6^{*}$ & $48.6 \pm 22.4$ & $0.43 \pm 0.12^{*}$ & $2.28 \pm 3.85^{*}$ \\
\hline $7(\mathrm{n}=165)$ & $6.27 \pm 4.83^{*}$ & $1.85 \pm 0.59^{*}$ & $11.62 \pm 5.47^{*}$ & $226.9 \pm 152.2$ & $50.16 \pm 58.3^{*}$ & $47.7 \pm 21.5$ & $0.43 \pm 0.13$ & $2.05 \pm 2.12$ \\
\hline $8(\mathrm{n}=126)$ & $6.29 \pm 5.16^{*}$ & $1.88 \pm 0.66^{*}$ & $10.99 \pm 5.61$ & $227.0 \pm 155.6$ & $58.5 \pm 67.9^{*}$ & $47.4 \pm 22.5$ & $0.44 \pm 0.14^{*}$ & $2.04 \pm 2.18^{*}$ \\
\hline$(\mathrm{n}=108)$ & $6.63 \pm 5.07^{*}$ & $2.01 \pm 0.71^{*}$ & $10.06 \pm 5.41$ & $201.3 \pm 131.5$ & $55.7 \pm 72.6$ & $48.1 \pm 25.3$ & $0.44 \pm 0.14^{*}$ & $2.36 \pm 3.03^{*}$ \\
\hline $10(\mathrm{n}=78)$ & $6.35 \pm 4.32^{*}$ & $1.89 \pm 0.54^{*}$ & $10.43 \pm 4.88$ & $202.4 \pm 125.9$ & $54.8 \pm 66.2$ & $43.4 \pm 17.1$ & $0.43 \pm 0.13^{*}$ & $2.11 \pm 2.49^{*}$ \\
\hline $11(n=56)$ & $5.76 \pm 4.44^{*}$ & $1.70 \pm 0.53^{*}$ & $11.26 \pm 4.69$ & $214.4 \pm 153.3$ & $59.0 \pm 91.6$ & $44.3 \pm 16.4^{*}$ & $0.42 \pm 0.14^{*}$ & $1.67 \pm 2.10^{*}$ \\
\hline $12(\mathrm{n}=17)$ & $6.82 \pm 6.51^{*}$ & $1.65 \pm 0.70^{*}$ & $10.06 \pm 5.57$ & $176.4 \pm 106.2$ & $94.6 \pm 117.8$ & $44.1 \pm 15.6^{*}$ & $0.40 \pm 0.13^{*}$ & $2.02 \pm 2.65$ \\
\hline
\end{tabular}

Values are presented as mean \pm standard deviation.

IPSS-T, total International Prostatic Symptom Score; QoL-I, quality of life index; Qmax, maximal flow rate; Vol, voided volume; PVR, postvoid residual; TPV, total prostate volume; TZI, transition zone index; PSA, prostate-specific antigen.

${ }^{*} \mathrm{P}<0.05$ compared with baseline.

Table 2. Reasons for discontinuing combination therapy according to different years of treatment duration

\begin{tabular}{lcccc}
\hline Reason & Total & $<2$ Years & $2-5$ Years & $>5$ Years \\
\hline Single medication: $\alpha$-blocker & $54(8.6)$ & $22(3.5)$ & $25(4.0)$ & $7(1.1)$ \\
a-blocker and antimuscarinics & $53(8.5)$ & $20(3.2)$ & $24(3.8)$ & $9(1.4)$ \\
Improvement of LUTS & $53(8.5)$ & $33(5.3)$ & $14(2.2)$ & $6(1.0)$ \\
Deceased & $52(8.3)$ & $35(5.6)$ & $13(2.1)$ & $4(0.6)$ \\
Surgical intervention & $39(6.2)$ & $19(3.0)$ & $14(2.2)$ & $6(1.0)$ \\
Single medication: antimuscarinics & $17(2.7)$ & $10(1.6)$ & $5(0.8)$ & $2(0.3)$ \\
LUTS symptoms persisted & $15(2.4)$ & $7(1.1)$ & $8(1.3)$ & $0(0)$ \\
High pill burden & $8(1.3)$ & $5(0.8)$ & $3(0.5)$ & $0(0)$ \\
Intermittency of medical consumption & $8(1.3)$ & $3(0.5)$ & $5(0.8)$ & $0(0)$ \\
Adverse effect & $6(1.0)$ & $3(0.5)$ & $1(0.2)$ & $2(0.3)$ \\
Loss of follow-up & $64(10.2)$ & $34(5.4)$ & $27(4.3)$ & $3(0.5)$
\end{tabular}

Values are presented as number of patients (\%).

LUTS, lower urinary tract symptoms.

tion of patients at each time point and the measured parameters at each time point are shown in Table 1. Interestingly, the 12year follow-up with combination therapy resulted in statistically significant continuous improvement in the IPSS-T and QoLI over all 12 years $(\mathrm{P}<0.018$ and $\mathrm{P}<0.001)$ respectively. Qmax, TPV, TZI, and PSA levels all showed significant improvement when compared to baseline levels in the first 4-8 years during the follow-up period.

\section{Causes of Discontinuation of Combination Therapy}

Among the 625 recruited patients, 369 patients (59\%) discontinued the combination medication. Among the 369 patients, 124 discontinued combination treatment due to changing medication to monotherapy with alpha-blockers only $(n=54$, $8.6 \%)$, antimuscarinics only ( $\mathrm{n}=17,2.7 \%)$, and combined alpha-blocker and antimuscarinics $(\mathrm{n}=53,8.5 \%)$. Thirty-nine patients (6.2\%) discontinued due to receiving surgical interven- 
tion, and 53 (8.5\%) due to improved LUTS. Only 64 (10.2\%) were loss to follow-up and $6(1.0 \%)$ discontinued combined treatment due to adverse effects (Table 2).

The treatment duration was divided into 3 groups: shortterm ( $<2$ years), middle-term (between 2 to 5 years) and longterm ( $>5$ years). In the short-term treatment duration group, the most common reasons for early termination of combination therapy were: deceased (35 patients, 5.6\%), loss to followup (34 patients, 5.4\%) and improvement of LUTS (33 patients, 5.3\%). In the middle-term treatment duration group, the most common reason was loss to follow-up (27 patients, 4.3\%), followed by conversion to a single medication with $\alpha-1$ blockers only (25 patients, $4.0 \%$ ), and conversion to $\alpha-1$ blockers and anti-muscarinic agents (24 patients, 3.8\%). However, in the long-term treatment duration group, most of the patients could adhere to combination therapy. The rates of medication conver-
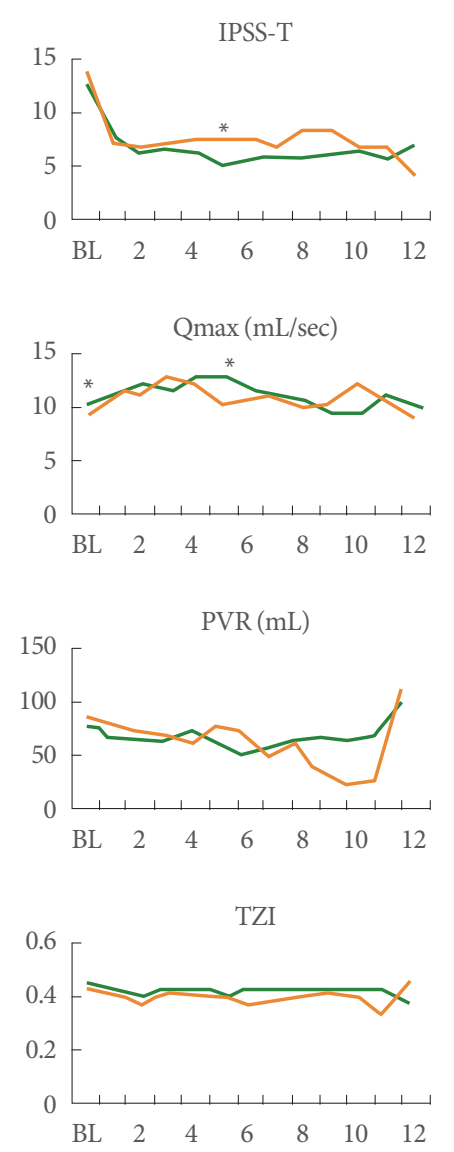

sion and surgical intervention were less than $2 \%$.

\section{Differences in Parameters Between the Discontinued and Continued Treatment Groups Over Time}

Over 12 years, there was consistent improvement in the IPSS and QoL-I. The most prominent improvement among all the parameters was in the first four years of follow-ups. There was no significant difference in the age between continued and discontinued treatment groups $(\mathrm{P}=0.484)$. Between continued and discontinued medication groups, most of the parameters showed no significant difference in time points of follow-up except in IPSS-T, QoL-I, Qmax, and Vol, in which the continued group had greater improvements at the 4 or 5 years of followup. However, TPV in the first and second year of follow-up was significantly smaller in the discontinued group compared to the continued group. The distribution of the measured parameters
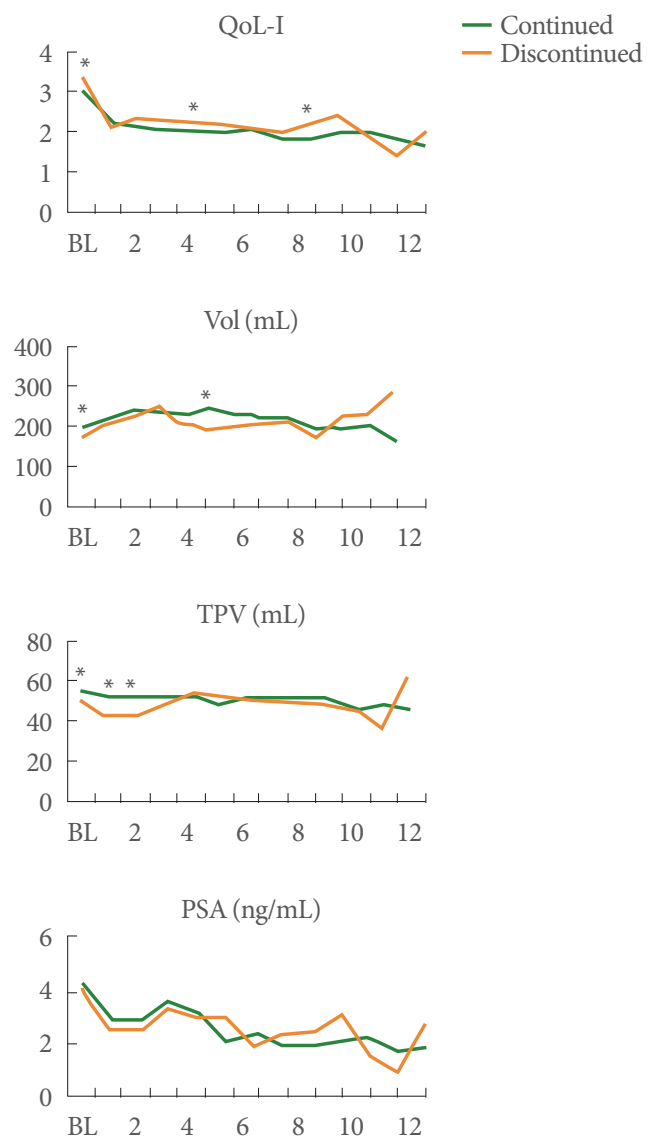

Fig. 1. The parameters in each year of follow-up between continued and discontinued medication groups. A paired t-test was used to compare variables between groups at each time-point. IPSS-T, total international prostatic symptom score; QoL-I, quality of life index; Qmax, maximal flow rate; Vol, voided volume; PVR, postvoid residual; TPV, total prostate volume; TZI, transition zone index; PSA, prostate-specific antigen; $\mathrm{BL}$, baseline. ${ }^{*} \mathrm{P}<0.05$ between 2 groups. 


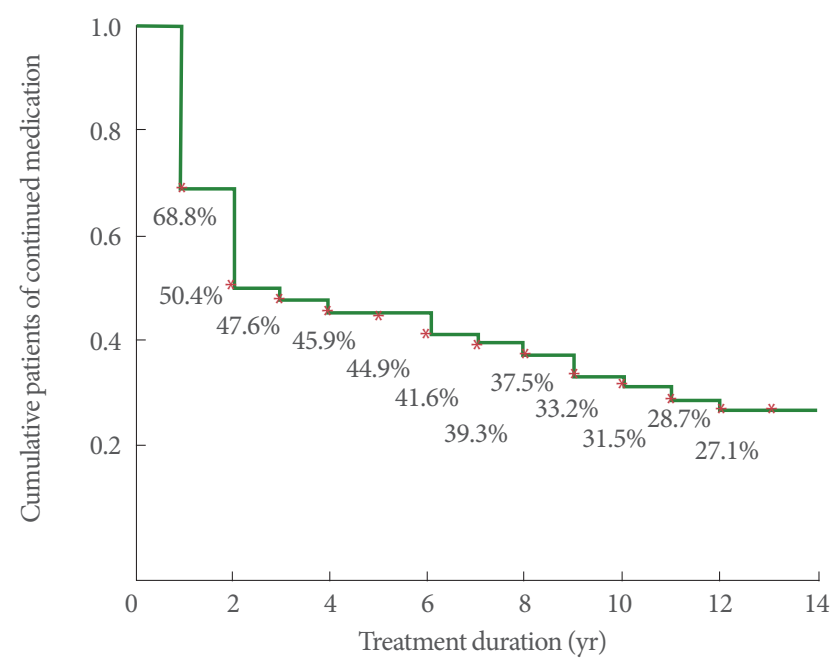

Fig. 2. Kaplan-Meier survival curve of patients who continued combination therapy.

between the 2 groups is shown in Fig. 1. After excluding 52 deceased patients, the remaining 573 patients were included in the analysis. The Kaplan-Meier survival curve of patients who continued combination therapy is shown in Fig. 2. There was a high drop-out rate in the first 2 years followed by a consistent but slow rate of withdrawal from combination treatment during the follow-up period.

\section{DISCUSSION}

This real-time, single hospital, prospective data collection and retrospective analysis study demonstrated significant improvements in all analyzed parameters, including TPV, TZI, PSA, Vol, PVR, Qmax, IPSS-T, and QoL-I. Interestingly, except in the cases of the IPSS-T and QoL-I, improvements in each variable did not consistently improve within the years of over 4 to 8 years of follow-up, indicating that dynamic characteristics of the prostate and flow rate parameters change over time.

$\mathrm{BPH}$ is one of the major factors leading to clinical LUTS. Elevation of PSA concentrations and TPV indicate deterioration of LUTS, and an increased risk of acute urinary retention and the need for surgical intervention [12,13]. TPV and PSA have become important parameters for further treatment. The CombAT study revealed that a combination of dutasteride and tamsulosin was more effective than monotherapy for the overall progression of LUTS in BPH [9]. The European Association of Urology guidelines currently show a benefit with 5ARI in improving LUTS in patients with moderate-to-severe LUTS and a
TPV > $40 \mathrm{~mL}$ or elevated PSA (>1.4-1.6 ng/mL) [11]. However, there are still no studies showing the long-term follow-up results of combination therapy in BPH patients. Previous studies have only included up to four years of follow-up [14]. The results of this study are comparable with the CombAT study. All measured variables improve after 4 years of combination therapy and only $1 \%$ of patients discontinue treatment due to surgical intervention, indicating a high patient compliance and tolerability to combination therapy. Moreover, our study showed that combination therapy provides effective and longlasting benefits over a long time span of more than 4 years.

Among the reasons for discontinued combination therapy, the most common reasons were due to changing medication and improved LUTS. The main reason for converting to a single medication was the presence of adverse effects due to 5ARI, such as sexual dysfunction (impotency, ejaculation problems) and gynecomastia [15]. Patients who changed from combined medication to antimuscarinics had reasons based on their main symptoms. Some patients who were bothered by both empty and storage LUTS in the beginning complained of residual storage LUTS after combined medication for a period of time. Because those patients had already been freed of empty LUTS, an antimuscarinic agent was prescribed. However, the patients who discontinued treatment due to changing medication decreased at the follow-ups for over 5 years, indicating that patients who are free of adverse effects, have good compliance to oral medication and improved LUTS usually can continue combination therapy for long time. The patients who converted to surgical intervention also decreased after more than 5 years of follow-ups, suggesting that patients who can adhere to combination therapy may not need BPH surgery because their condition is stable.

Regarding the treatment duration, this study showed that the mean duration of combination therapy was 4.6 years in patients who adhered to the combination treatment, but only 2.2 years in those who discontinued combination therapy. The most important factor of discontinued combination therapy in our results was due to adverse effects that contributed to converting to monotherapy. This finding was similar to that of previous trials, where higher discontinuation rates were observed in the combination therapy group compared to the monotherapy group at the first 2-year follow-up [16].

Because the mean age in this study was older than 70 years, with an improvement of IPSS and QoL-I compared to the baseline, patients tended to discontinue combination therapy espe- 
cially in the first 2 years of follow-up. This indicated that the patient might have already felt satisfied with the effects of combination therapy and tended to stop medication by themselves. This is why a high percentage of patients discontinued treatment in under 2 years and at the 2- to 5 -year follow-up. Subjective satisfaction with combination therapy is an important factor in choosing long-term medical treatment with combined medications. The result clearly shows patients' concerns and strongly influenced adherence to the combination therapy.

Although TPV was weakly correlated with BPH symptoms and QoL-I, TPV was considered to be a predictor for long-term medical adherence [17]. In this study, we found that patients with a smaller TPV tended to discontinue the combination therapy within the first 2 years. These patients might also have improved LUTS in addition to a smaller TPV after short-term combination therapy. Therefore, they might discontinue combination therapy when their symptoms improve. Some patients with small TPV might not benefit from combination therapy and request surgical intervention or a change in medication to relieve their severe LUTS. A good patient-doctor relationship and education of the benefits of long-term combination therapy for $\mathrm{BPH}$ is very important in contributing to better adherence to combination therapy [18].

Limitations of this study are no placebo control group, no double-blind study, uncontrolled prescription of alpha-blockers, and large follow-up data loss. However, in real world practice, patient loss during a long-term treatment period is expected. Improvement of patient education may increase patient compliance to medical treatment of $\mathrm{BPH}$.

In conclusion, combination therapy of an $\alpha-1$ blocker and 5ARI lead to significant improvements over baseline in LUTS, uroflowmetry parameters, and prostate variables over time. This was observed not only in the short-term but also over a long-term follow-up period of 12 years. Changing medications, improved LUTS and choosing surgery are common reasons for discontinuing combination therapy. A larger TPV after shortterm combination treatment was among the factors that causes the withdrawal from combination therapy.

\section{ACKNOWLEDGEMENTS}

I would like to express my thanks to Professor Kuo and Dr. Liao for the discussions, which helped me develop the ideas, put forward here.

\section{REFERENCES}

1. Jacobsen SJ, Girman CJ, Lieber MM. Natural history of benign prostatic hyperplasia. Urology 2001;58(6 Suppl 1):5-16.

2. Emberton M, Fitzpatrick JM, Garcia-Losa M, Qizilbash N, Djavan

B. Progression of benign prostatic hyperplasia: systematic review of the placebo arms of clinical trials. BJU Int 2008;102:981-6.

3. Emberton M, Cornel EB, Bassi PF, Fourcade RO, Gómez JM, Castro R. Benign prostatic hyperplasia as a progressive disease: a guide to the risk factors and options for medical management. Int J Clin Pract 2008;62:1076-86.

4. Djavan B, Marberger M. A meta-analysis on the efficacy and tolerability of alpha1-adrenoceptor antagonists in patients with lower urinary tract symptoms suggestive of benign prostatic obstruction. Eur Urol 1999;36:1-13.

5. Lepor H, Williford WO, Barry MJ, Haakenson C, Jones K. The impact of medical therapy on bother due to symptoms, quality of life and global outcome, and factors predicting response. Veterans Affairs Cooperative Studies Benign Prostatic Hyperplasia Study Group. J Urol 1998;160:1358-67.

6. Roehrborn CG, Boyle P, Bergner D, Gray T, Gittelman M, Shown T, et al. Serum prostate-specific antigen and prostate volume predict long-term changes in symptoms and flow rate: results of a fouryear, randomized trial comparing finasteride versus placebo. PLESS Study Group. Urology 1999;54:662-9.

7. Gormley GJ, Stoner E, Bruskewitz RC, Imperato-McGinley J, Walsh PC, McConnell JD, et al. The effect of finasteride in men with benign prostatic hyperplasia. The Finasteride Study Group. N Engl J Med 1992;327:1185-91.

8. McConnell JD, Roehrborn CG, Bautista OM, Andriole GL Jr, Dixon CM, Kusek JW, et al. The long-term effect of doxazosin, finasteride, and combination therapy on the clinical progression of benign prostatic hyperplasia. N Engl J Med 2003;349:2387-98.

9. Roehrborn CG, Siami P, Barkin J, Damião R, Major-Walker K, Nandy I, et al. The effects of combination therapy with dutasteride and tamsulosin on clinical outcomes in men with symptomatic benign prostatic hyperplasia: 4-year results from the CombAT study. Eur Urol 2010;57:123-31.

10. McVary KT, Roehrborn CG, Avins AL, Barry MJ, Bruskewitz RC, Donnell RF, et al. Update on AUA guideline on the management of benign prostatic hyperplasia. J Urol 2011;185:1793-803.

11. Oelke M, Bachmann A, Descazeaud A, Emberton M, Gravas S, Michel MC, et al. EAU guidelines on the treatment and follow-up of non-neurogenic male lower urinary tract symptoms including benign prostatic obstruction. Eur Urol 2013;64:118-40. 
12. Emberton M, Fitzpatrick JM, Rees J. Risk stratification for benign prostatic hyperplasia (BPH) treatment. BJU Int 2011;107:876-80.

13. Madersbacher S, Marszalek M, Lackner J, Berger P, Schatzl G. The long-term outcome of medical therapy for BPH. Eur Urol 2007;51: 1522-33.

14. Roehrborn CG, Lukkarinen O, Mark S, Siami P, Ramsdell J, Zinner N. Long-term sustained improvement in symptoms of benign prostatic hyperplasia with the dual 5alpha-reductase inhibitor dutasteride: results of 4-year studies. BJU Int 2005;96:572-7.

15. Descazeaud A, de La Taille A, Giuliano F, Desgrandchamps F, Doridot G. Negative effects on sexual function of medications for the treatment of lower urinary tract symptoms related to benign prostatic hyperplasia. Prog Urol 2015;25:115-27.
16. Cindolo L, Pirozzi L, Fanizza C, Romero M, Tubaro A, Autorino R, et al. Drug adherence and clinical outcomes for patients under pharmacological therapy for lower urinary tract symptoms related to benign prostatic hyperplasia: population-based cohort study. Eur Urol 2015;68:418-25.

17. Liu CC, Wang CJ, Huang SP, Chou YH, Wu WJ, Huang CH. Relationships between American Urological Association symptom index, prostate volume, and disease-specific quality of life question in patients with benign prostatic hyperplasia. Kaohsiung J Med Sci 2004;20:273-8.

18. Koh JS, Cho KJ, Kim HS, Kim JC. Twelve-month medication persistence in men with lower urinary tract symptoms suggestive of benign prostatic hyperplasia. Int J Clin Pract 2014;68:197-202. 